\title{
Cemento-Ossifying Fibroma of the Mandible: A Case Report Including Its Imaging and Histopathological Features
}

\author{
Misha Efney Fernandes*, Surekha R Puranik \\ Department of Oral Medicine and Radiology, P.M. Nadagouda Memorial Dental College and Hospital, Bagalkot, India
}

Received May 8, 2021; Revised June 10, 2021; Accepted July 19, 2021

\section{Cite This Paper in the following Citation Styles}

(a): [1] Misha Efney Fernandes, Surekha R Puranik, "Cemento-Ossifying Fibroma of the Mandible: A Case Report Including its Imaging and Histopathological Features, "Open Journal of Dentistry and Oral Medicine, Vol. 9, No. 1, pp. 1 - 6, 2021. DOI: 10.13189/ojdom.2021.090101.

(b): Misha Efney Fernandes, Surekha R Puranik (2021). Cemento-Ossifying Fibroma of the Mandible: A Case Report Including its Imaging and Histopathological Features. Open Journal of Dentistry and Oral Medicine, 9(1), 1 - 6. DOI: 10.13189/ojdom.2021.090101.

Copyright $\bigcirc 2021$ by authors, all rights reserved. Authors agree that this article remains permanently open access under the terms of the Creative Commons Attribution License 4.0 International License

\begin{abstract}
Cemento-ossifying fibroma is the uncommon benign fibro-osseous lesion of the craniofacial skeleton. The origin of this lesion has been traced to the periodontal ligament since the cells can form cement, lamellar bone, or fibrous tissue. The lesion most often occurs in the jaws, especially the mandible. Most lesions are small and incidentally found. Larger lesions cause facial asymmetry or functional impairment. The lesion is more common in females. Of the craniofacial bones, the mandible is the most commonly affected site, with the lesion typically inferior to the premolars and molars. From a radiological perspective, some lesions can be exceptionally radiopaque, or some well-defined with mixed or unilocular radiolucency. A 23-year-old female presented with a swelling in the right mandibular region, radiographs revealed a mixed radiopaque - radiolucent lesion showing a centrifugal expansion. Computed tomography revealed a mixed predominantly opaque expansile lesion with massive buccolingual expansion. Complete excision of the lesion was performed and histopathology findings confirmed it as Cemento-Ossifying Fibroma. Through this case report we want to signify the importance of diagnosis, consecutive therapy after clinical and radiographic findings, as such lesions continue expanding and may lead to a severe medical complication. The knowledge of the different types of radiological findings is important. The unique radiographic appearance of the lesion, in this case, can be
\end{abstract}

beneficial to help clinicians understand. Due to the good delimitation of the lesion surgical removal and curettage is the treatment of choice. Complete surgical resection of the lesion is essential to achieve good results in terms of health and recurrence.

Keywords Mandible, Fibroma, Ossifying, Panoramic, Radiography

\section{Introduction}

Cemento-Ossifying Fibroma is a type of fibro-osseous lesion and behaves like a benign osteogenic tumor. The lesion shows a predilection for females, having a female to male ratio of 4:1. It manifests as asymptomatic, slow-growing, and well-demarcated. Asymmetrical deformation of face, aesthetical and functional impairment results as a consequence of prolonged and slow growth of the tumor [1]. The lesion is more frequently reported in the second to fourth decades of life, and usually involves the mandible. Radiographic features depend on the duration of the lesion, initially well-defined radiolucent, later mixed radiolucent-radiopaque with opacities in the middle of the lesion and as the lesion matures, it appears with asymmetrical opacities forming concentric bony trabeculae with marginal sclerosis [2]. The pathogenesis 
remains unknown; it may be related to problems in the maturation of dental tissue mostly cementum and bone arising from the ectopic periodontal ligament or mesodermal germ layer or mesenchymal cells [1-3]. The histopathology of the tumor consists of highly cellular, fibrous tissue consisting of variable amounts of bone, cementum, or both. The treatment of choice is surgical excision with extended resection that depends on the size and location of the lesion [1,2]. This study aimed to describe its course on clinical, radiological, and histopathological characteristics of the tumor.

\section{Case Report}

A 23-year-old female reported to our department with a chief complaint of swelling in the right mandibular region for 6 months. The swelling was not associated with pain or paraesthesia over the region. The patient did not have any significant past dental and medical history. Extra-orally a solitary diffuse swelling was seen over the right lower one-third region of the mandible. Overlying skin appeared to be normal. On palpation, it was bony hard in consistency, fixed and non-tender, with no other changes on manipulation. Intra orally a dome-shaped swelling of $1.5 \times 1.5 \mathrm{~cm}$ extending from the lingual gingiva aspects of 44,45,46 and mesial part of 47, intact stretched mucosa with lingual and buccal vestibular obliteration. It was bony hard, non-tender, fixed with cortical expansion from 44 to 46 region (Fig 1). Teeth were vital on electric pulp testing and a mesial displacement of 45 was noted.

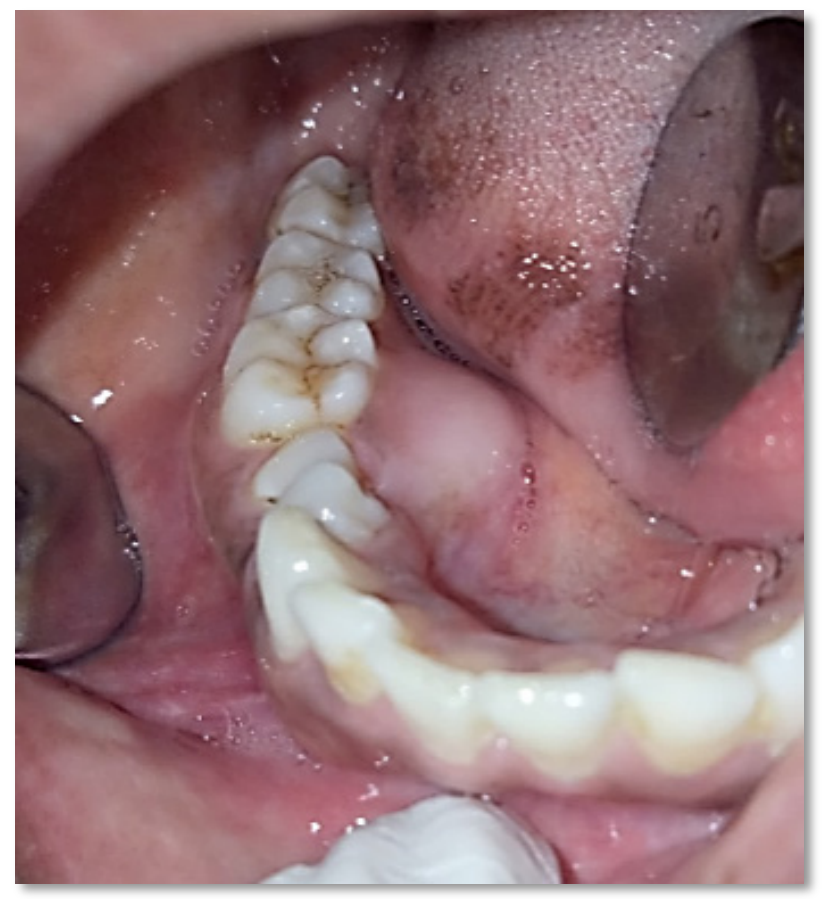

Figure 1. Lingual cortical expansion with intact mucosa
Radiographically, the intraoral periapical radiograph (IOPA) showed homogeneously dense radiopaque shadow from 44 to mesial aspect of 47 , obscuring the shadows of roots of $44,45,46$. Displacement of 45 was noted as seen in the IOPA. (Fig 2 a). The occlusal radiograph showed an expansile radiopaque shadow having a centrifugal expansion pattern. Tufts of amorphous dense cementum-like radiopacities with a flocculent pattern were seen internally with intact outer cortical border (Fig 2 b).

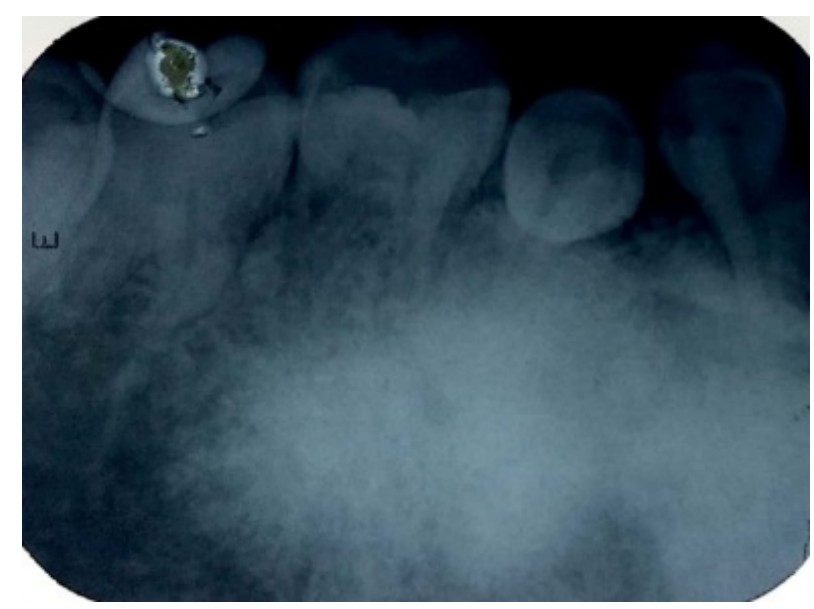

Figure 2a. IOPA showing homogenously dense radiopacity

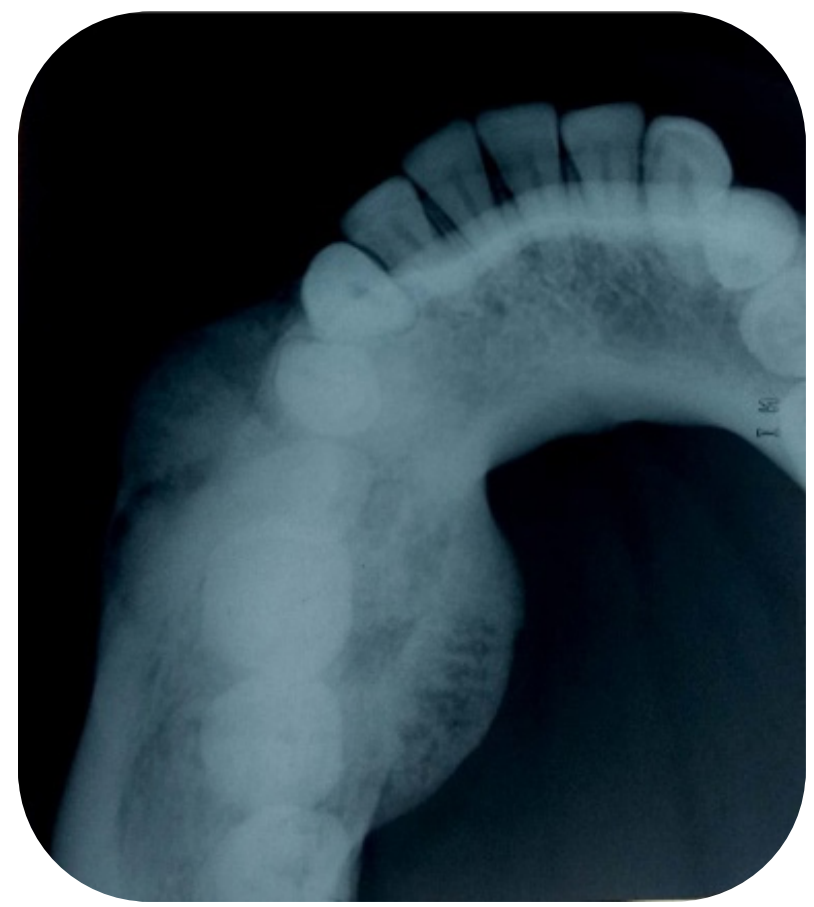

Figure 2b. Occlusal radiograph showing amorphous dense radiopacity and centrifugal expansion

The Panoramic radiograph revealed a mixed lesion in the right body of the mandible about $3.5 \times 2 \mathrm{~cm}$, extending from the cervical crestal region of 44 to 47 , obscuring the roots 44 to 46 , causing inferior displacement of the inferior alveolar canal. The lesion was in-homogeneously 
radio-opaque in the anterior aspect and radiolucent with subtle radiopaque flecks near the $2^{\text {nd }}$ molar with indistinct border. 44,45 teeth were lingually displaced. Indistinct radiolucent band seen at the periphery of the lesion (Fig 3).

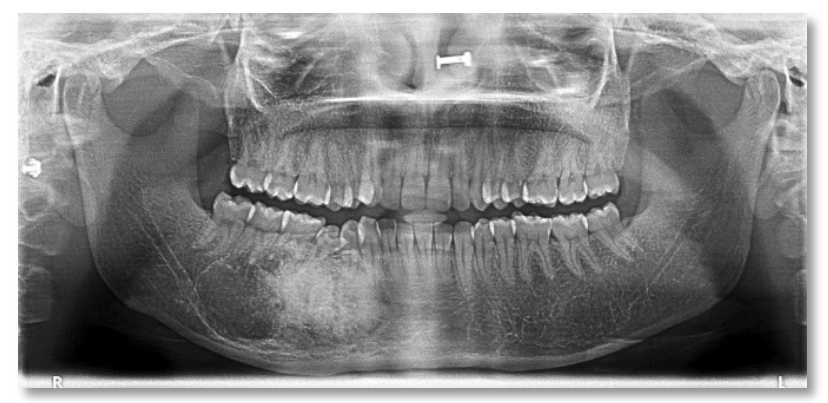

Figure 3. Panoramic radiograph revealing mixed lesion with radiolucent rim

Computed Tomography (CT) Scan showed well-defined mixed predominantly opaque expansile lesion in the right body of mandible with massive buccolingual expansion. An internally amorphous flocculent pattern having cementum like radiodensity (Fig 4). 3 D CT revealed a lobulated lesion with intact cortex, no perforation on the buccal and lingual aspects. (Fig 5)
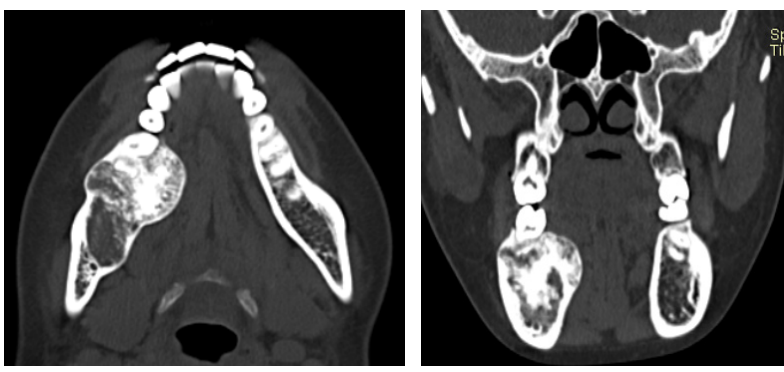

Figure 4. CT scan; A coronal section, B axial section showing inhomogeneous mixed predominantly radiopaque, buccolingual expansion, internally amorphous flocculent pattern
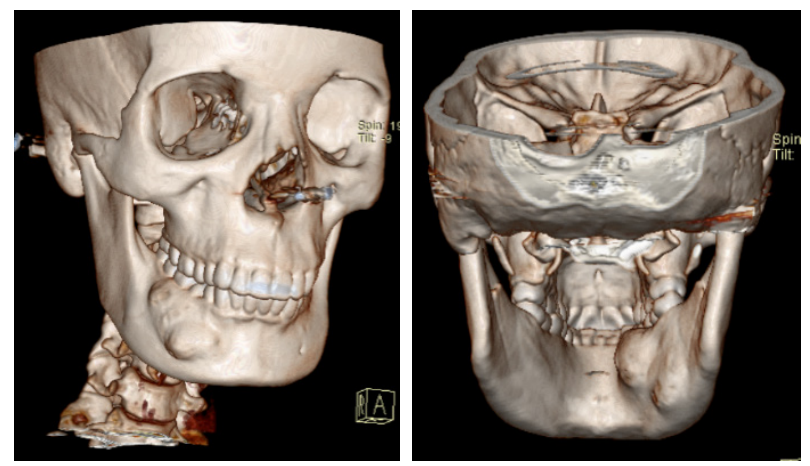

Figure 5. 3 D CT showing lobulated lesion with intact cortex, no perforation on buccal and lingual aspect

The lesion was surgically excised and curettage of the area was performed. The histopathology revealed cellular rich lesional tissue consisting of spindle to stellate shaped cells arranged in the form of short whorls and fascicles.
Numerous round to oval shaped, of varying sized basophilic areas with rough texture, that were separated by an empty space from the stromal cells suggestive of cementum. (Figure 6) These findings along with clinic-radiological observation confirmed the diagnosis as Cemento-ossifying fibroma. The patient was subjected to clinical and radiological examination after excision of the lesion to discard any recurrence.

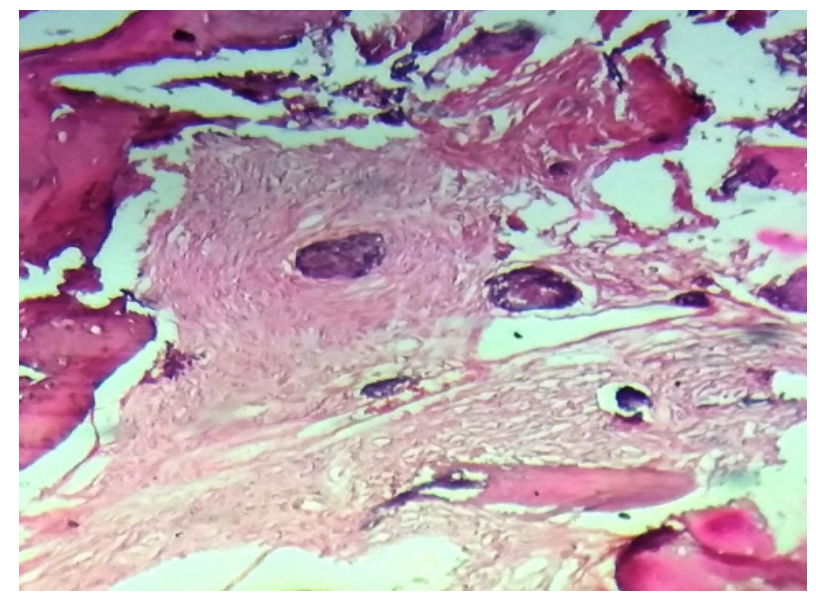

Figure 6. Histopathology showing rich fibrous connective tissue and cementum

\section{Discussion}

Cemento-ossifying fibroma is a benign fibro-osteogenic tumor with membranous ossification, hence exclusively involving the maxillofacial region [3]. A variant of ossifying fibroma termed as Cemento-Ossifying Fibroma was described by Menzel [4] in 1872. Montgomery [5] in 1927 reported the first case and he gave the name as Ossifying Fibroma. There are different schools of thought regarding the etiology of this lesion, which may be traumatic or developmental or a result of mesenchymal induction $[1,5,6]$. It has been proposed that trauma or dental extraction may be a stimulating factor for the periodontal cells attached to the walls of the alveolus [6]. Recent microscopic studies of the lesion in the other maxillofacial region led to the conclusion of its origin from the mesenchymal cells within the mesodermal germ layer and resulted in a complex tumor. Some of the ossifying fibromas arise from the periodontal ligament, which contains pluripotential cells capable of forming cementum, bone, and fibrous tissue [7,9]. However; some authors raise a controversy on this concept because of lesions arising in the bone lacking periodontal ligaments like ethmoid bone, or frontal bone [1]. Some categorize this lesion as a localized dysplastic change in the bone metabolism process. Others have attributed it to neoplastic nature because of its aggressive behaviour leading to bone destruction [9]. It generally occurs between the second and fourth decade, with a 1:4 male: female ratio. The most 
frequent location is mandibular, involving the premolar and molar region [1]. It has a region-wise predilection in the order of molar region (52\%), followed by the premolar $(25 \%)$, incisor (12\%), and cuspid regions (11\%) [16]. The neighbouring tissues can be pushed with or without being destroyed. Depending on its degree of mineralization, it is more or less firm in consistency. Clinically, the lesion presents as a painless, slow-growing, intra bony mass leading to teeth displacement, and is covered with normal mucosa. These features were also noted in our case $[1-3,6]$.

World Health Organization has categorized the term "Cemento-Ossifying Fibroma" as a type of mesenchymal odontogenic tumor. The predominance of bone in the lesion is termed as ossifying fibroma; if cementum is present, it is termed cementifying fibroma, and the presence of both bone and cementum are termed as cemento- ossifying fibroma [6,11]. There are two variants of cemento - ossifying fibroma; the central variant arising from the cells of the periodontal ligament in the apical area resulting in expansion of lamina dura and; the peripheral variant occurs in the soft tissues of the teeth-bearing areas [6]. There have been more aggressive lesions reported characterized by rapid and extensive growth often leading to fracture of the mandible. Similarly, an aggressive juvenile form has been described as a juvenile cemento-ossifying fibroma $[1,8]$.

The characteristic radiographic feature of cementoossifying fibroma is expansion, lesion margination, demarcation, or cortication [5,12]. Lesion is always related to the periapical region of the teeth. A few cases have been reported extending to the angle- ramus area or encroaching the maxillary sinus. The radiographic finding of cemento-ossifying fibroma is a well-demarcated unilocular or multilocular ovoid or round-shaped, lesion with smooth contours [5]. The radiographic appearance is of utmost importance for diagnosis. Three radiographic patterns are described depending on its borders: defined lesions without a sclerotic border or with a sclerotic border or ill-defined border [10]. The lesion follows a different degree of mineralization. Initially presenting as an osteolytic image and transforming into mixed and in some cases as completely radiopaque. The radiopaque foci type, shows a pattern similar to that seen in fibrous dysplasia or like a wispy pattern (similar to stretched tufts of cotton) or a flocculent pattern (similar to large heavy snowflake) $[4,8]$. Important diagnostic feature is reflected in the growth of the lesion, which tends to be concentric within the medullary part of the bone with outward expansion equal in all directions also referred to as centrifugal growth pattern $[4,5]$. The tumor maintains a spherical shape, expands without cortical perforation, and may lead to tooth divergence [1,4]. Typical ossifying fibroma grows in centrifugal fashion producing ball-like circular lesion. Lesion tends to enlarge equally in all directions producing expansion of buccal and lingual cortical plate and notably the inferior cortex; as seen in our case. The expanded inferior cortex tends to be parallel to the margin of the tumor mass. Larger lesions of the mandible often demonstrate a characteristic downward bowing of inferior cortex of mandible [5]. The mandible expansion leads to displacement of mandibular canal-like; this feature is noted in our case. The lamina dura of involved teeth usually is missing, and resorption of teeth may occur $[1,7]$.

In computed tomography, a well-differentiated mass shows the same density as a bone, centre, showing low density [3]. The lesion is best imaged on computed tomography and sometimes a magnetic resonance imaging may be indicated for surgical planning or to evaluate complication. Computed tomography of cemento-ossifying fibroma exhibits a thin intact cortex. Imaging features vary depending on the amount of fibrous and bony tissue within the lesion. In the early stages, there is the presence of a thick peripheral rim of the bone surrounding a fibrous soft-tissue centre: It was suggested that in the centre of the lesion there is a progressive deposition of mature bone in the later stages $[3,13,14]$. Signal intensity on magnetic resonance imaging is dependent upon the amount of bone trabeculae and degree of cellularity. Ossifying fibroma usually has low to intermediate signal on T1- weighed images. The lesion shows a hyperintense central region or a predominantly hyperintense lesion. The hypointense signal intensity on T2-weighed images is caused by numerous bony trabeculae. MRI offers greater specificity where there is neurovascular and ocular involvement [13].

Histopathologic examination of cemento-ossifying fibroma shows a proliferation of irregularly shaped calcifications within a hypercellular fibrous connective tissue stroma. The calcification pattern appears differently and reflects the various stages of bone and cementum deposition. The most common pattern is of small osteoid trabeculae surrounded by osteoblasts [5,15]. Histologic differentiation between osteoid and cementum is difficult. Histologically the lesion shows proliferation of irregularly shaped calcification within a hypercellular fibrous connective tissue stroma $[3,10]$. According to Woo et al [8] the spindle fibroblast-like proliferation that deposits cementicles and osteoid and woven bone, often with prominent osteoblastic rimming. Pieces of a fibrous capsule can be present [8]. Generally, histopathological examination shows a hypercellular fibroblastic stroma with collagen fibers, blood vessels, and variable amounts of mineralization. The stromal cells range from spindle-shaped to polyhedral with large prominent basophilic nuclei and scant cytoplasm. In certain cases, cells are tightly packed with a fascicular or storiform pattern [15]. Under polarized light, early tumors show a woven bone pattern, and mature lesions show trabeculae of lamellar bone. Some lesions also contain ovoid calcified structures resembling 'cementicles' or 
psammoma bodies; these are dense, calcifies and basophilic may even show a concentric layer of mineralization. The fibrous tissue can have a storiform or pinwheel pattern with fine irregular calcification resembling the pattern of dystrophic bone [15].

The differential diagnosis with fibrous dysplasia particularly with cementifying variant is complicated because morphology in both cases is similar. In Fibrous dysplasia, ossification is abnormal and irregular with no peripheral osteoblast. Several histopathological sections of the lesion need to be carefully examined since in every section the findings are different. Lesions appearing as a mixed periapical image like that of calcifying odontogenic cysts or cementoblastomas; these are related to roots of vital teeth. Focal cemento-osseous dysplasia appears as radiolucent associated to tooth apex and in advanced stages appears as radiopaque with a radiolucent halo. Histological examination is difficult at early stages of the disease, hence multiple sections have to be always studied and examined thoroughly. During resection, the absence of clear limits between the lesion and healthy bone helps to differentiate between Cemento-ossifying fibroma. Other lesions like periapical osteocementum dysplasia and cemento-ossifying fibroma can occur in the apical region of the vital tooth. Cemento-ossifying fibroma of the mandible goes through stages of maturation, similar to those of periapical osteocementum dysplasia, in older patients predominately smaller in the region of incisors and canines. Cemento-ossifying fibroma with mixed radiographic features can be misdiagnosed as a calcifying odontogenic cyst or adenomatoid tumor. Other differential diagnosis of cemento-ossifying fibroma with radiopaque radiographic features includes retained tooth, odontoma, idiopathic osteosclerosis, condensing osteitis, or osteoblastoma $[1,3,16]$.

The management of cemento-ossifying fibroma depends on its clinical and radiological presentation and usually entails one of the following methods; enucleation, curettage or resection. Complete resection of the lesion and curettage is the treatment of choice since the lesion is well encapsulated and has a good prognosis since recurrences are not reported frequently. Small lesions must be treated conservatively by curettage or enucleation until a healthy margin is reached. Larger lesions require a complete radical surgical section. Cemento-ossifying fibroma are well encapsulated hence they shell out easily at surgery; difficulty occurs in the maxillary lesion than the mandibular lesion. This has been attributed to the difference in bone texture. The recurrence rate of mandibular cemento-ossifying fibromas varies from 6 to $28 \%$. The recurrence of the maxillary lesion is unknown, but is likely higher because of surgical removal difficulties encountered $[1-4,6]$.

\section{Conclusions}

Cemento-ossifying fibroma is an uncommon benign fibro-osseous tumor of the craniofacial region. The diagnosis of cemento-ossifying fibroma is vital; oriented by clinical, radiological, and histopathological aspects. Mostly cosmetic and dental problems are the first manifestation. The diagnosis in the initial stage can be often confused with periapical pathologies due to its radiolucent appearance. The clinical presentation of the lesion is painless and expansive; it can grow to giant size involving vital structures. Due to its hybrid nature, the entire lesion must be excised and several histopathologic sections should be examined. Early detection and complete surgical enucleation must be employed with a subsequent follow up.

\section{Glossary of Abbreviation}

IOPA - Intraoral periapical radiograph

CT - Computed Tomography

3 D CT - 3-Dimensional Computed Tomography

$\mathrm{cm}$ - Centimetre

\section{Conflicts of Interest}

None.

\section{REFERENCES}

[1] Rangil JS, Silvester FJ, Requeni-Bernal J., "Cemento-ossifying fibroma of the mandible: Presentation of a case and review of the literature," J Clin Exp Dent, vol. 3, no. 1, pp. 66-69, 2011. DOI:10.4317/jced.3.e66

[2] Titinchi F, Morkel J, "Ossifying fibroma: Analysis of treatment methods and recurrence patterns," J of Oral Maxillofac Surg, vol.74, no. 12, pp. 2409-2419,2016. DOI: 10.1016/j.joms.2016.05.018

[3] Trijolet JP, Parmentier J, Sury F, Goga D, Mejean N, Laure B, "Cemento-ossifying Fibroma of the mandible. Eur Ann of Otorhinolaryngol Head and Neck Dis," vol. 128, no. 1, pp. 30-33, 2011. DOI: 10.1016/j.anorl.2010.06.005

[4] Gondivkar SM, Gadbail AR, Chole R, Parikh RV, Balsaraf $\mathrm{S}$, "Ossifying fibroma of the jaws: Report of two cases and literature review," Oral Oncol. vol. 47, no. 9, pp. 804-809, 2011. DOI: 10.1016/j.oraloncology.2011.06.014

[5] Agarwal N, Gupta P, Gupta P, Naik S, Upadhyay N, "Recurrent bimaxillary radiopacities: A rare case report. Contemp Clin Dent," vol. 3, no. 1, pp. 103-108,2012. DOI: 10.4103/0976-237X.95117.

[6] Aburas S, Bandura P, Al- Ibraheem A, Berger S, Meier M, Turhani D, "A Large maxillary Cemento-Ossifying Fibroma superimposed with Solitary Bone Cyst 
Documented Over 18 Years: A Case Report,” Int J Surg Case Rep, vol. 68, pp. 257-262, 2020. DOI: 10.1016/j.ijscr.2020.03.011

[7] Liu Y, Wang H, You M, Yang Z, Miao J, Shimizutani K, Koseki T, "Ossifying fibromas of the jaw bone: 20 cases Dentomaxillofacial Radiology," vol. 39, no. 1, pp 57-63, 2010. DOI: $10.1259 / \mathrm{dmfr} / 96330046$

[8] Woo SB, "Central Cemento-Ossifying Fibroma: Primary Odontogenic or Osseous Neoplasm?," J Oral Maxillofac Surg, vol. 73 , no. 12, pp 87-93,2015. DOI: 10.1016/j.joms.2015.05.010.

[9] Triantafillidou K, Venetis G, Karakinaris G, Iordanidis F, "Ossifying fibroma of the jaws: a clinical study of 14 cases and review of the literature," Oral Surg Oral Med Oral Pathol Oral Radiol, vol. 114, no. 2, pp. 193-199, 2012. DOI: 10.1016/j.tripleo.2011.07.033

[10] Barberi A, Cappabianca S, Colella G, "Bilateral Cemento-Ossifying fibroma of maxillary sinus," $\mathrm{Br} \mathrm{J}$ Radiol, vol. 76, no. 904, pp. 279-280, 2003. DOI: 10.1259/bjr.76.904.760279

[11] Wright JM, Vered M, "Update from the 4th Edition of the World Health Organization Classification of Head and Neck Tumours: Odontogenic and Maxillofacial Bone
Tumors," Head Neck Pathol, vol. 11, no. 1, pp. 68-77, 2017. DOI: $10.1007 / \mathrm{s} 12105-017-0794-1$.

[12] Makek MS, "So called "fibro-osseous lesions" of tumorous origin. Biology confronts terminology," J Craniomaxillofac Surg, vol. 15, no. 3, pp. 154-167, 1987. DOI: 10.1016/s1010-5182(87)80040-9

[13] Salina ACI, Souza PMM, Gadelha CMDC, Aguiar LB, Castro JDV, Barreto ARF, "Ossifying fibroma: an uncommon differential diagnosis for T2-hypointense sinonasal masses," Radiol Case Rep, vol. 7, no. 12, pp. 313-317, 2017. DOI: 10.1016/j.radcr.2017.03.019.

[14] McCollister KB, Hopper BD, Michel MA, "Sinonasal neoplasms: update on classification, imaging features, and management," Appl Radiol, vol. 44, no. 12, pp. 7-15, 2015.

[15] Zegalie N, Speight PM, Martin L, "Ossifying fibromas of the jaws and craniofacial bones," Diagnostic Histopathology," vol. 21, no. 9, pp. 351-358, 2015. DOI: http://dx.doi.org/10.1016/j.mpdhp.2015.07.004

[16] Chang CC, Hung HY, Chang JY, Yu CH, Wang YP, Liu $\mathrm{BY}$, Chiang $\mathrm{CP}$, "Central ossifying fibroma: a clinicopathologic study of 28 cases," J Formos Med Assoc, vol. 107, no. 4, pp. 288-294, 2008. DOI: 10.1016/S0929-6646(08)60089-3. 